\title{
R-CHOP versus CHOP in HIV-associated lymphoma: a meta-analysis of prospective studies
}

\author{
Ignacio Echenique ${ }^{1}$, Jorge Castillo ${ }^{2^{*}}$ \\ From 13th International Conference on Malignancies in AIDS and Other Acquired Immunodeficiencies \\ (ICMAOI) \\ Bethesda, MD, USA. 7-8 November 2011
}

\begin{abstract}
Introduction
Several recent trials have demonstrated superiority with the addition of rituximab to traditional chemotherapeutic regimens in HIV-negative non-Hodgkin lymphoma (NHL) patients. In HIV-positive patients, the benefit of adding rituximab is less clear. In a randomized controlled trial, the addition of rituximab to CHOP showed no survival advantage. We performed a study-level meta-analysis of prospective studies to ascertain outcomes in HIV+ NHL patients treated with $\mathrm{CHOP}$ vs. R-CHOP.
\end{abstract}

\section{Methods}

We performed a Pubmed/MEDLINE literature search from January 1990 through June 2011, with search terms "(HIV OR AIDS) AND lymphoma AND rituximab” and limited our results to English language prospective trials with either CHOP or R-CHOP in HIV+ NHL. Characteristics and outcomes were collected from published data. Chisquare was used to compare the characteristics between groups. The main outcomes were overall response rate (ORR), complete response (CR) rate and 2-year overall survival (OS) and will be reported as odds ratio (OR).

\section{Results}

We identified 3 studies on HIV+ NHL patients treated with $\mathrm{R}-\mathrm{CHOP}$ and 9 with $\mathrm{CHOP}$ from a total of 119 publications. Nine studies (75\%) administered Pneumocytis jirovecci pneumonia prophylaxis. Four studies (33\%) administered prophylactic intrathecal chemotherapy, and in 3 (25\%) it was optional. Four studies (33\%) administered G-CSF routinely, and 3 studies (25\%) only if grade $3 / 4$ neutropenia occurred. A total of 810 patients were studied, 569

\footnotetext{
* Correspondence: jcastillo@lifespan.org

${ }^{2}$ Division of Hematology and Oncology, The Warren Alpert Medical School of Brown University, The Miriam Hospital, Providence, RI, USA

Full list of author information is available at the end of the article
}

treated with $\mathrm{CHOP}$ and 241 with $\mathrm{R}-\mathrm{CHOP}$. The median age was 38 and 43 years for $\mathrm{CHOP}$ and $\mathrm{R}-\mathrm{CHOP}$, respectively, with $86 \%$ and $85 \%$ of male patients, respectively $(\mathrm{p}=0.98)$. With regard to HAART, $68 \%$ of patients treated with $\mathrm{CHOP}$ and $92 \%$ with R-CHOP were on HAART prior to lymphoma diagnosis $(\mathrm{p}<0.0001)$. The median CD4 count was 109 and 136 cells $/ \mathrm{mm}^{3}$ in CHOP and R-CHOP patients, respectively. Clinically, $65 \%$ and $54 \%$ of $\mathrm{CHOP}$ patients presented with advanced stage and age-adjusted International Prognostic Index (aaIPI) score 2-3, while the proportion was $74 \%$ and $45 \%$ in $\mathrm{R}-\mathrm{CHOP}$, respectively ( $\mathrm{p}=0.02$ for stage and $\mathrm{p}=0.03$ for aaIPI scores). The OR for ORR, CR and 2-year OS in patients treated with R-CHOP vs. CHOP was 1.05 (95\% CI 0.71-1.55; $\mathrm{p}=0.81), 1.42$ (95\% CI 1.04-1.93; $\mathrm{p}=0.03)$ and 2.37 (95\% CI 1.73-3.25; $\mathrm{p}<0.0001)$, respectively.

\section{Conclusions}

HIV + NHL patients treated with R-CHOP had higher odds for CR and 2-year OS (42\% and 137\%, respectively) when compared to CHOP. However, patients treated with R-CHOP also had higher rates of HAART administration, higher CD4 counts and lower aaIPI scores.

\section{Author details}

'Department of Medicine, The Warren Alpert Medical School of Brown University, The Miriam Hospital, Providence, RI, USA. ²Division of Hematology and Oncology, The Warren Alpert Medical School of Brown University, The Miriam Hospital, Providence, RI, USA.

Published: 19 April 2012

doi:10.1186/1750-9378-7-S1-P22

Cite this article as: Echenique and Castillo: R-CHOP versus CHOP in HIVassociated lymphoma: a meta-analysis of prospective studies. Infectious Agents and Cancer 2012 7(Suppl 1):P22.

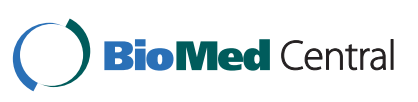

() 2012 Echenique and Castillo; licensee BioMed Central Ltd. This is an open access article distributed under the terms of the Creative Commons Attribution License (http://creativecommons.org/licenses/by/2.0), which permits unrestricted use, distribution, and reproduction in any medium, provided the original work is properly cited. 